\title{
Induction of Sensory Organs by the Uninvaginated Portion of the Dorsal Lip of the Amphibian Gastrula.
}

\author{
By Yô K. OKADA and Tadao HAMA. \\ Zoological Institute, Faculty of Science, Tokyo University. \\ (Comm. by I. AMEMIYA, M.J.A., Dec. 13, 1948:)
}

When the uninvaginated portion of the dorsal lip of the blastopore at the crescentic groove stage, stage III of gastrulation according to Okada and Hama $(1943)^{1)}$, was implanted in the forepart of a young neurula and covered over with the presumptive ectoderm of an advanced gastrula that had practically finished invaginating - this experimental method was employed especially when the induction of sensory organs was desired,-in almost every instance (9 out, of 10) it underwent differentiation into notochord together with cartilage or muscle, and provoked the induction of ear (see figures). The development of ear took place not only in the presence of hindbrain (fig, 1) but also in its absence (figs. 2 and 3). Indeed, the latter condition ( 6 instances out of 10) occurred far more frequently than the former ( 3 instances out of 10 ) so that otic formation may not be said to be necessarily dependent upon the action of the hindbrain. It seems rather to be attendant on the differentiation of the transplant into notochord and to be induced directly by notochord, the existence of hindbrain being a secondary matter.2 The connection between notochordal differentiation of the dorsal lip and otic induction was likewise observed when, instead of the aforementioned procedure, the dorsal lip was wrapped in presumptive ectoderm from an advanced gastrula. In this experiment the specimens, one example being shown in figure 4, lacked both cartilage and muscle. To be sure, that the uninvaginated part of the dorsal lip should undergo such differentiation and bring about such induction seems to be the natural outcome from our knowledge of its prospective fate (cf. Okada and Hama, 1945b) ${ }^{3}$, but this fact was true not only of the uninvaginated dorsal lip of stage III, but also of that of almost all the gastrulur stages both before and after stage III. Thus, in an earlier investigation of Okada and Hama $(1943)^{4}$, notochordal differentiation was accomplished by 85 per cent of the test material from stage I, by 94 per cent of that from stage II, and by 100 per cent of that from subsequent stages. In comparison, the frequency of otic

1) Proc. 19, 48.

2) Compare with Harrison, R.G. (1935, '38); Holtfreter J. ('35); Waddington, C.H. ('37).

3) Proc. 21, 342.

4) Proc. 19, 50. 
induction was 60 per cent or more for tissue of all stages from I'through VII with the exception of stage VI. Here, the failure to attain the minimum frequency was in all probability caused by the scarcity of samples. In this same experiment, for the reason that the ectoderm employed was derived from a very young gastrula at the onset of invagination, ear rarely occurred alone, being accompanied

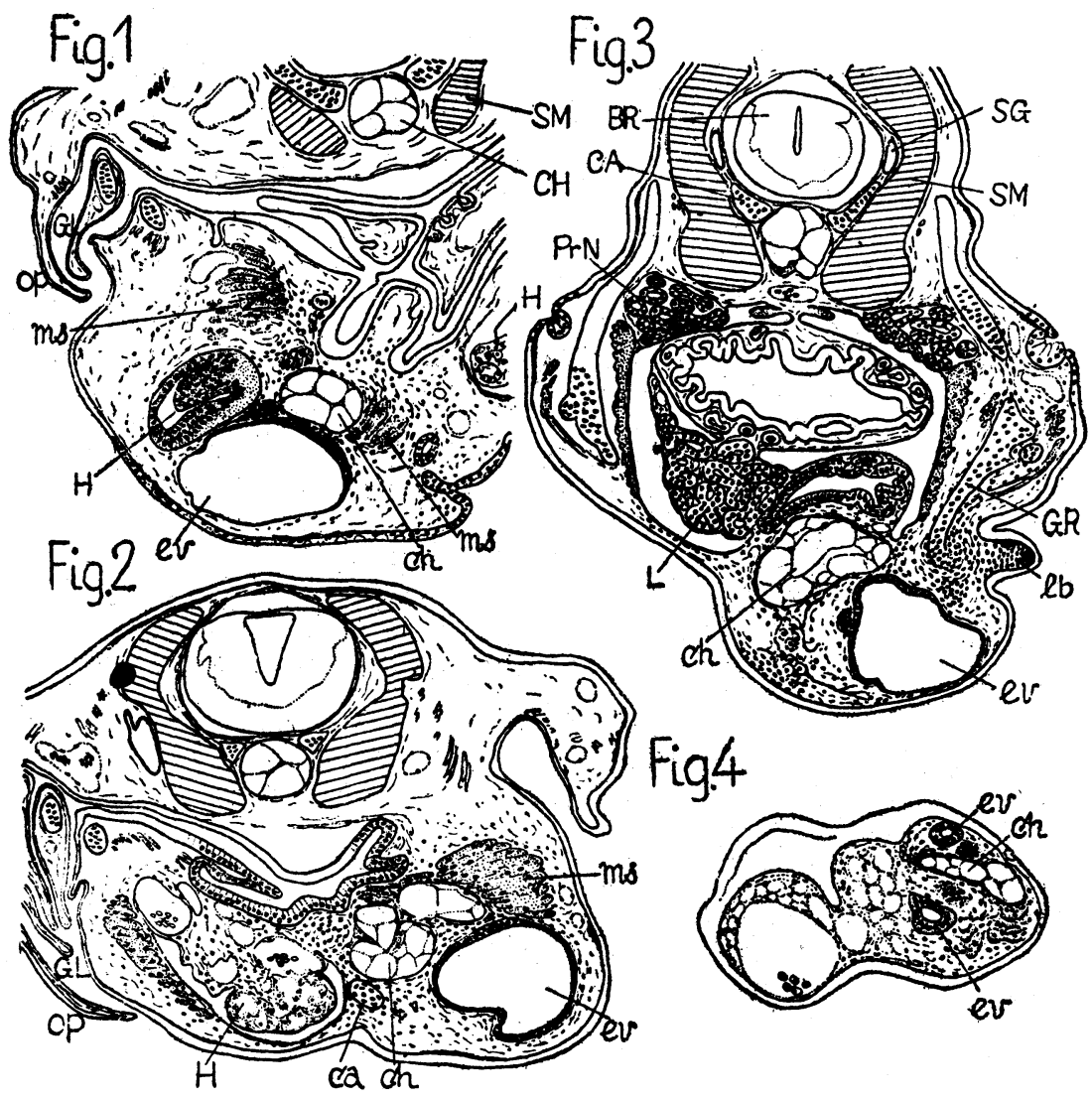

Some examples of implantation (fig. 1-3) and culture (fig. 4) of uninvaginated portion of dorsal blastoporal lip at crescentic groove stage. (Capital letters represent organs of the host)

BR (br) brain, ca cartilage, ch notochord, ev ear vesicle, GL gill, GR girdle,

$H$ heart, L liver, lb limb, ms muscle, OP operculum, $\operatorname{PrN}$ pronephros, SG spinal ganglion, SM somite.

usually by brain and frequently, in the case of organizer tissue of stages through stage $V$, by preotic sensory organs, such as nose and eye. At any rate, the differentiation into notochord of the dorsal blastoporal lip of stages prior to stage III and the induction of ear by the dorsal lip of stages other than III are not in keeping with the prospective fate of the explanted tissue. 
Here to be mentioned are the results of Kawakami (1943), ,") who has worked with the same end in view as the authors. Kawakami divided the period of gastrulation into twelve stages, designating the appearance of the ventral lip of the blastopore as stage X. In testing the ability of the uninvaginated dorsil lip of each gastrular stage to induce eephalic sensory organs, he obtained results essentially the same as those described above. As far as the uninvaginated "dorsial lip of stages corresponding to stage IV and later of Okada and Hama was concerned, provided that the implant survived, it differentiated into notochord, and in case of stages IV and V it often in tuced ear in the absence of brain. The former outcome was secured in 9 out of 22 instances ( $41 \%$ ), and the latter, in 7 . out of $19(37 \%)$. With material from subsequent stages, however, although the differentiation of the transplant into notochord manifested even greater regularity, the induction of ear took a sudden drop, being realized just once in 8 cases of stage VI and in 5 of stage VII respectively. At a glance, it may seen that this fact should be compared to the regional differentiation of the inductive capacity of the dorsal wall of the archenteron, set forth by Okada and Hama (1945a), ${ }^{\text {(5) }}$ and should be regarded as anticipating the evolvement of the regional differences. On the other hand, in the aforementioned experiment of culturing the dorsal lip in a vesicle of ectoderm from a young gastrula, such a decrease in the percentage of otic induction did not occur even in the ciase of stage VII (see 1.c., p. 50, table 1). It is not impossible that here, the difference in experimental methods may have been the cause of the reduction. When employing the dorsal border of the blastoporal remnant after gastrulation, Kawakami secured ear in 67 per cent (4 out of 6 ) of the sjecimens. In any case the fact that differentiation into notochord and induction of ear was achieved by the tested tissue of such a late stage and, as in Okilda and Hama's experiment by the dorsal lip of stages earlier than stage III, neither of which includes material destined to become notochord, is certainly a situation that deserves due consideration.

It should be noted, nevertheless, that in assembling the results, Kawakami treated the divisions made by Okada and Hama in the gastrulation process up through stage III more or less as one; consequently, in as much as a part of the notochordal material is.already included in the uninvaginated dorsal lip of stage III, his results are not absolute with respect to the above question. Even so, among 26 specimens as many as $\mathbf{1 6}$ displayed notochordal differentiation, of

5) 植動 (Botany and Zoology) 11, 859.

6) Proc. 21, 240.

7) Proc. 19, 50. 
which 12 had also croked otic induction. Moreover, aside from ear, only mouth and gills were inciucer-and these in only one instance each,- - while other sensory organs were totally lacking. Judged from the writers' results concerning the regionial alifierentiation of the inductive caparity of the archenteric roof-and Kawak:mi is of the same mind,- - the foregoing group of tests ought to have producerl the induction of nose and lens as well. Therefore, the experiment was repeated, carc being taken to select the dorsal blastoporal lip at the beginning of gastrulition in order to avoid the rossible inclusion of notochordal material. The outeome still showed differentiation into notochorl in 5 of the 10 cases of grafts from stige $I,{ }^{8}$ but an indubitable induction of ear in not more than 1 case. Of the 5 fitiling to exhilit notochordal differentiation, 3 became unorganized masses of endodermal cells, next to which cartilage developed. The latter was likewise apjarent with notochord. Furtherhore, out of the total number there were $\mathbf{3}$ instances of brain induction, one of which, though small, was of extremely complex strunture. Upon close inspection this particular brain was found to be possessed of a nose on its frontal surface; a vesicular structure, thought to be optic vesicle, on either side; and two lenses amid the nasal tissue. At first glance, however, it gave the impression of beifg nothing more than an aggregation of neural tissue because of its poor general development and its confinement in a small spacc. Not far from this brain a pair of small vesieles were also detected which ware assumed to be otic vesicles. Thus, in these tests, too, with tissue obtained from the first gastrular stage and destined to grow into foregut, differentiation into notochord was evident in half of the specimens; induction of brain was also noted, but that of ear was rare. In the experimental group using material of stige II, however, inductions of ear were more numerous. Accordingly, one may innagine a period in the course of devclopment of the preotie substratum, during which its tissue is able to effect differentiation into notochord and induction of hindbrain and ear. Subsequently it undergoes a change of its differentiation so that its anterior end evolves into endolerm and its posterior end into mesolerm. A simultaneous change of its inductive capacity occurs so that it becomes al)le to induce forebrain and preotic sensory orgins. Hence, the single instance, imperfect though it was, of formation of preotic sensory organs by the transplanted dorsal lip of stage I may be thought of as an early manifestation of the said change of indurtire capacity. At the same time, there is the fact, brought to light by the investigation dealing with the regional differentiation of

8) The blastopore is only indicated by pigments accumulating where invagination will be initiatcd. 
the inducing capacity of the dorsal wall of the archenteron, that the inducing ability of this initially invaginating portion is lost with time (cf. Okada and Hama, 1945a, p. 246)."

Under the circumstances, when and how does the dorsal lip of each stage acquire the ability to induce in accordance with its prospective fate? Past experience points to invagination as the time of origin, the morphological changes taking place then being accompanied by important histological changes. In support of this view is Woerdeman's $(1933)^{10)}$ phenomenon of glycolysis. Also, Hama (unpublished) has observed the differential staining of eytoplasms with thionin, those of uninvaginated cells being dyed blue and those of invaginated cells, red. Nevertheless, the distinction perceived by these two investigators between uninvaginated and invaginated parts characterized the dorsal lip of all gastrular stages; therefore, they can scarcely be thought to distinguish between notochord and head mesoderm or between otic and preotic areas. Thus, much additional research is still necessary to establish for certain the responsibility for the regional differences in the inductive capacity of the dorsal lip and of the archenteric roof and to substantiate further the attendant chemical differcences of the various parts At the same time, in this connection one fact has been made known up to the present; namely, the ready loss by both dorsal lip and archenteric roof of the regional differences in their inductive capacity following the application of heat, individuals often appearing with simply lens in the absence of notochordal differentiation and otic induction. For example, when Kawakami $(1943)^{11)} \mathrm{im}$ mersed pieces of the uninvaginated dorsal lip of the blastopore and of the doral wall of the arehenteron in hot water of $60^{\circ}$ to $65^{\circ} \mathrm{C}$ for 2 to 3 minutes, he obtained inductions of the so-called free lens in ectoderm from late gastrular stages in at least 1 out of 15 instances.

9) Proc. 21, 240.

10) Kon. Akad. Wetensch. Amsterdam Proc., 36, 189.

11) 動雜 (Dobutsu Gaku Zassi) 55, 359. 